\title{
Dentistry and OMICS: Transcriptome Dynamics of an Oral Ecosystem as Measured by Changes in Oral Polymorphonuclear Neutrophils in Experimental Gingivitis
}

\author{
Patrick Rijkschroeff,, Ton Schoenmaker, ${ }^{1}$ Martien Caspers, ${ }^{2}$ Lars Verschuren, ${ }^{2}$ Bart J.F. Keijser, ${ }^{2-4}$ \\ Elena A. Nicu, ${ }^{1,5}$ and Bruno G. Loos ${ }^{1}$
}

\begin{abstract}
Oral health and dentistry are essential components of systems medicine, which has received lesser attention in comparison to other medical fields, such as cancer biology. In this context, oral polymorphonuclear neutrophils (oPMNs) play an important role in the maintenance of oral health. To the best of our knowledge, this is the first study to report original observations on the transcriptional responses of oPMNs during experimentally induced gingivitis, by temporarily refraining from regular oral care. Oral rinses were prospectively collected at four different time points for oPMNs isolation from healthy volunteers: day 1 (start of the experimental gingivitis challenge), day 9 (during challenge), day 14 (end of the challenge), and day 21 (postchallenge). Transcriptome of oPMNs was determined by RNA sequencing. Differentially expressed genes (DEGs) were selected at $p<0.01$ level, and evaluated for pathway regulation using Ingenuity Pathway Analysis suite. We found four major clusters of DEGs, consisting of 256 initial response DEGs (day 9 only), 221 late response DEGs (day 14 only), 53 persistent responsive DEGs (consistent at day 9 and 14), and 524 DEGs showing responses only in the postchallenge phase (day 21 only). Pathway analysis of the initial and late response DEGs showed involvement in many immune regulatory pathways and PMN function, whereas DEGs at day 21 were associated with epithelial adherence signaling and other miscellaneous related signaling pathways. The results from this pilot study showed that oPMNs mediate oral inflammatory processes, suggesting their immunomodulatory role in oral equilibrium.
\end{abstract}

Keywords: dentistry, oral health, gingivitis, transcriptomics, neutrophils

\section{Introduction}

$\mathbf{P}$ OLYMORPHONUCLEAR NEUTROPHILS (PMNs) are essential in the elimination of microbial infections. PMNs typically extravasate from a nearly sterile blood circulation toward infected sites of inflammation and sites of tissue damage. When PMNs are released from the bone marrow, they are considered as fully differentiated cells and have traditionally been regarded as transcriptionally inactive
(Stejskal et al., 2010). Only a few studies have therefore shown their interest in analyzing transcriptional responses of PMNs in vivo (Calvano et al., 2005; de Kleijn et al., 2012).

Interestingly, it was previously shown that RNA expression patterns in PMNs can change dramatically when exposed to bacteria (Subrahmanyam et al., 2001). In any microorganism-enriched environment, such as the oral cavity, PMNs are able to migrate toward various chemoattractants and are recruited to the frontline where the oral

\footnotetext{
${ }^{1}$ Department of Periodontology, Academic Center for Dentistry Amsterdam (ACTA), University of Amsterdam (UVA) and Vrije Universiteit Amsterdam (VU), Amsterdam, The Netherlands.

${ }^{2}$ Microbiology and System Biology, TNO Earth, Environmental and Life Sciences, Zeist, The Netherlands.

${ }^{3}$ Department of Preventive Dentistry, Academic Center for Dentistry Amsterdam (ACTA), University of Amsterdam and Vrije Universiteit Amsterdam, Amsterdam, The Netherlands.

${ }^{4}$ Top Institute Food and Nutrition, Wageningen, The Netherlands.

${ }^{5}$ Opris Dent SRL, Sibiu, Romania.
} 
mucous tissues and the gingival crevices interact with an invasion of many microorganisms (Delima and Van Dyke, 2003; Dutzan et al., 2016). From here, PMNs are able to exert their antimicrobial functions like phagocytosis and neutrophil extracellular trap (NET) formation and are then recognized as oral PMNs (oPMNs) (Moonen et al., 2019).

Functionally active oPMNs have been studied in oral health, in gingivitis and in periodontitis. As an example, during gingivitis and periodontitis, the oral cavity is populated with increased numbers of oPMNs (Bender et al., 2006; Landzberg et al., 2015). A recent review described that many studies have observed an altered functional behavior for oPMNs ex vivo in relation to oral health and oral inflammation (Hirschfeld, 2019). Moreover, it has been suggested that oPMNs may therefore play a role in the initiation and progression of oral inflammatory diseases, such as gingivitis and periodontitis (Aboodi et al., 2011; Matthews et al., 2007).

However, it is currently not fully understood to what extent the oPMNs contribute to immune regulation in the oral cavity. Since PMNs are classically implicated in inflammation, but have resolution properties also, it seems highly likely that the oPMNs are able to play a role in oral immunity through microbial surveillance and other means of antimicrobial activity, such as phagocytosis and NET formation (Chapple and Matthews, 2007; Moutsopoulos and Konkel, 2018; Silvestre-Roig et al., 2016; White et al., 2016).

The current study investigated early inflammatory mechanisms within the oral cavity using the human experimental gingivitis model. This is a commonly accepted and welldescribed model to study the initiation and resolution of gingival inflammation, since it is universally applicable in individual subjects and considered appropriate due to its rapidly inducible and reversible nature (Löe et al., 1965; Wahaidi et al., 2011). We aimed to study the role of oPMNs during the initiation and resolution of experimentally induced gingivitis by evaluating their gene expression dynamics.

In this study, we present the transcriptional responses of oPMNs when the oral ecosystem changes during the course of a 2-week experimentally induced gingivitis challenge, followed by a 1-week postchallenge "resolution phase" in healthy volunteers. By characterizing the oPMNs' transcriptome, we aimed to identify changes in gene expressions within the experimental period, thereby gaining knowledge on the oPMNs' functioning in relation to the development and resolution of gingival inflammation.

\section{Materials and Methods}

A prospective cohort study was conducted at the Academic Center for Dentistry Amsterdam (ACTA) between February and June 2015 (van der Veen et al., 2016). This study was performed within the framework of the TI Food and Nutrition project "An exploratory study on the dynamic (microbial, biochemical, and immunological) interactions of the oral ecosystem during induction of mild gingival inflammation; Dynamics of a Healthy Oral Ecosystem". The clinical trial was approved by the Medical Ethics Committee of the VU University Medical Center, The Netherlands (2014.505) and registered at the public trial register of the Central Committee on Research Involving Human Subjects under trial number NL51111.029.14. This study was carried out in accordance with the recommendations of Good Clinical Practice guide- lines (World Medical Association, 2013) and the Medical Research Involving Human Subjects Act.

All subjects were informed about the purpose of the study, received written information, and had given written consent before inclusion, in accordance with the Declaration of Helsinki.

\section{Study participants}

oPMN gene expression was studied in healthy volunteers who were recruited and screened at ACTA for eligibility. Healthy male and female volunteers between 18 and 55 years of age were included if they did not participate in another clinical study in the previous 30 days (van der Veen et al., 2016). Systemically healthy subjects (as assessed by a medical questionnaire) were included with a minimum of 20 natural teeth, including the presence of the first and second molars. Furthermore, all participants had a dental check-up within 1 year before the start of the clinical trial, and confirmed having finished any necessary dental treatment needed. Dental students and employees from ACTA were excluded from participating.

All subjects were screened for their periodontal condition using the Dutch Periodontal Screening Index (DPSI; Van der Velden, 2009); included patients presented with DPSI scores of $\leq 3$ - in any sextant and $<40 \%$ full mouth bleeding (van der Veen et al., 2016).

Volunteers with untreated dental caries, removable partial dentures, night guards, (peri-)oral piercings, apparent oral lesions, and the presence of orthodontic appliances (except lingual retention wires) were excluded from this study. Further exclusion criteria were: smoking, exsmokers (refrained for a period shorter than 1 year), pregnancy or breastfeeding women, use of antibiotics during the last 3 months, need of antibiotic prophylaxis before dental treatment, use of antiinflammatory drugs on a regular basis or any adverse medical history of (long-term) medication (except for contraceptives), and volunteers with self-reported abuse of drugs or alcohol.

\section{Study procedures}

For the purpose of the study regarding "Dynamics of a Healthy Oral Ecosystem", each participant was randomly assigned to either an intervention or a control group. Our research questions of this present cohort study do not include this intervention group, and therefore the intervention arm of the study was excluded from the present study.

Before the start of the gingivitis challenge, participants were monitored when performing their normal oral hygiene routine at day 14 (pre-challenge). All volunteers were then instructed to refrain from any form of oral hygiene for a period of 2 weeks (days 1-14), starting $24 \mathrm{~h}$ before the first appointment at the start of the experimental gingivitis challenge (day 1). During these 2 weeks of plaque accumulation, an additional visit was planned at day 9. At the end of the challenge, the habitual oral hygiene routine was reestablished by each participant. The final visit planned in the study was at day 21. At the onset of this postchallenge "resolution phase" (day 14-21) all participants were provided with a standardized manual toothbrush, fluoride toothpaste, and specific instructions to reinitiate manual brushing.

A flowchart showing the study design is presented in Figure 1. All participants were instructed not to eat and drink (with the exception of water) $2 \mathrm{~h}$ before any study appointment. 


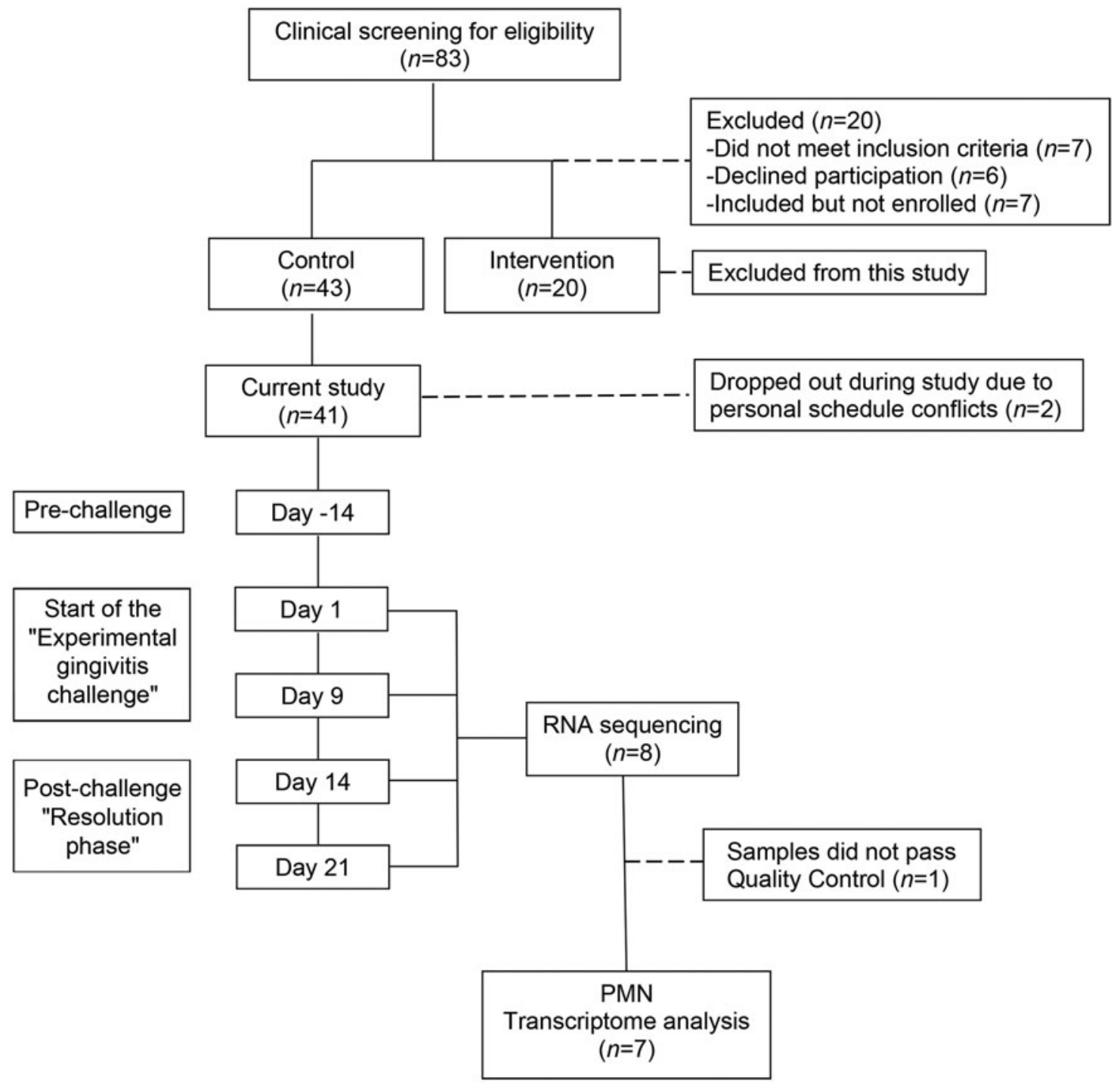

FIG. 1. Flowchart of the experimental setup of the study. Of the 63 volunteers enrolled in the parent study, 61 subjects completed the entire study (Van der Veen et al., 2016). For the purpose of the current study, samples were first assessed for quality (RNA concentration and RNA integrity). Next, eight subjects were selected from the control group, based on the yield of oPMNs and the amount and purity of the RNA samples. Samples from eight subjects were sequenced, which revealed two outliers belonging to one subject and therefore did not pass the quality control. The oPMN transcriptome from seven subjects were suitable for further analyzes. oPMN, oral polymorphonuclear neutrophil.

\section{Dental plaque and gingival bleeding assessment}

The extent of dental plaque development and gingival bleeding were clinically measured for the purpose of the study, "Dynamics of a Healthy Oral Ecosystem". The complete protocol and the results of plaque and bleeding assessments are described in a previous study (van der Veen et al., 2016). In short, dental plaque was assessed in a half mouth randomized contralateral model. A modified Silness and Löe Plaque Index (Silness and Löe, 1964) was used on a four-point scale (0-3) to evaluate six sites of the buccal and lingual aspects of all present teeth (Van der Weijden et al., 1993) at days 1, 14 and 21.

One independent calibrated examiner measured dental plaque levels throughout the experiment. Another independent calibrated examiner measured gingival bleeding levels throughout the experiment. Gingival bleeding was evaluated in a half mouth randomized contralateral model for bleeding on marginal probing (BOMP) areas (Lie et al., 1998). A BOMP index was used on a three-point scale (0-2) and a BOMP score was given to six gingival areas of the buccal and lingual sides of all present teeth at days 0,14 and 21. Percentages were calculated by dividing the number of sites with dental plaque or gingival bleeding by the total number of sites.

\section{OPMN isolation}

For each participant, oral rinses were collected at four time points (days $0,9,14,21)$. The oPMNs were isolated on the same day directly after oral rinse sampling. The collection procedure was based on a previously described protocol (Rijkschroeff et al., 2016). Briefly, five serial rinses were obtained from the oral cavity of each participant between 
09:00 and 12:00. Volunteers were instructed to rinse thoroughly with $5 \mathrm{~mL}$ of sterile sodium chloride solution $(0.9 \%$ $\mathrm{NaCl}$ ), each time for $30 \mathrm{sec}$ with a $3 \frac{1}{2} 2 \mathrm{~min}$ intermission, and were instructed neither to gargle nor to clear their throat during the sampling procedure. The pooled expectorated samples were kept on ice in a $50 \mathrm{~mL}$ centrifuge tube (SigmaAldrich, Zwijndrecht, The Netherlands) until the end of the collection procedure.

Oral rinses were filtered through a series of nylon mesh filters (Merck Millipore, Darmstadt, Germany); $70 \mu \mathrm{m}$ followed by $30 \mu \mathrm{m}$ and $10 \mu \mathrm{m}$. The filtered samples were centrifuged at $500 \mathrm{~g}$ for $10 \mathrm{~min}$ at $4^{\circ} \mathrm{C}$. The supernatant was discarded and the pelleted cells were resuspended in phosphate buffered saline (Gibco ${ }^{\circledR}$, Paisley, United Kingdom) and kept on ice for cell quantification and RNA isolation. Cells were quantified with a Muse ${ }^{\circledR}$ Cell Analyzer (Merck Millipore) using the Counts and Viability kit according to the manufacturers' instructions.

\section{$R N A$ isolation}

The oPMNs were suspended in RNA lysis buffer (Zymo Research, Irvine, CA, USA), and stored at $-80^{\circ} \mathrm{C}$ until RNA isolation. Total RNA was isolated using the quick-RNA mini prep kit (Zymo Research) according to the manufacturers' protocol, without DNAse treatment. Samples were shipped to Baseclear (Baseclear, Leiden, The Netherlands), where RNA concentration and RNA integrity numbers were assessed using the Agilent 2100 BioAnalyzer.

\section{RNA sequencing}

RNA sequencing was performed on the Illumina $\mathrm{Hi}_{-}$ Seq2500 system according to the Baseclear protocol. Briefly, after mRNA purification using polyA capture, library preparations were made using the TruSeq library prep kit. Singleend sequence reads were generated using the Illumina HiSeq2500 system and FASTQ sequence files were generated using the Illumina Casava pipeline version 1.8.3. The quality of the FASTQ sequences was enhanced by trimming off low-quality bases using the CLC Genomics Workbench version 8.5.1. Sequencing results were mapped to the GRCh38.p7 human genome assembly (Gene expression omnibus, GSSE118304).

\section{Data analysis and statistical analysis}

Gene expression data was normalized per subject over time by dividing the readouts for raw counts per gene per sample by the readouts for the total counts per sample, multiplied by the maximum counts per sample of all samples, followed by $\log$ transformation. Unexpressed genes were removed from further analysis. Differentially expressed genes (DEGs) compared with day 1 expression levels (start of the experimental gingivitis challenge), were used as an input for pathway analysis through Ingenuity Pathway Analysis suite (http://ingenuity.com). The calculated $p$-values $<0.01$ were used as threshold for significance, which indicates the significance of the overlap between the known target genes of a transcription factor and the same DEGs measured in the experiment at a different time point. Repeated Measures analysis of variance was performed. Mean and standard deviations (SDs) were calculated where appropriate.

\section{DESeq quality analysis}

The aim of this study was to evaluate whether transcriptional responses of oPMNs occur at the start, during the development, and after resolution of experimentally induced gingivitis. For this purpose, samples from initially eight subjects were subjected to transcriptomic analyses and RNA sequencing was performed on 32 samples (Fig. 1). All counts were mapped on the human reference genome resulting in total of 14,881 mapped genes in the oPMNs after DESeq2 normalization. RNA expression from oPMNs in three different time points (day 9, 14, 21) were analyzed and compared with the gene expressions at day 1 .

Quality control analysis of the samples, revealed two outliers based on the sample distance matrix and intensity distribution (Supplementary Fig. S1). These two samples belonged to one subject in particular and included day 1 and 14 data (start of the challenge and endpoint challenge). For this reason, the data belonging to this subject were removed from further analyses. Differential gene expression analysis was then based on 28 samples originating from seven subjects (Supplementary Fig. S2).

\section{Results}

\section{Study population}

Before the start of the study "Dynamics of a Healthy Oral Ecosystem", 83 potential participants were screened for eligibility. In total, 41 volunteers from the control group completed the entire trial (Fig. 1). Eight subjects from this group were selected on the basis of a steady-state oPMN cell count, for the purpose of prospectively characterizing the changes in the oPMN transcriptome by RNA sequencing.

A total of 32 samples at four time points from eight individuals were analyzed to determine the DEGs relative to expression levels at the start of the experimental gingivitis challenge. Based on the quality analyses of RNA analyses, one subject was excluded, yielding a final study population of seven subjects (Fig. 1). These seven subjects were all females (25.2 years \pm 7.1 ; mean/SD).

\section{Dental plaque and gingival bleeding levels indicate a proinflammatory response during experimental gingivitis}

The results of dental plaque levels and BOMP are presented in Figure 2. The percentage of sites with dental plaque or gingival bleeding was calculated. Dental plaque levels accumulated during the experimental gingivitis challenge, which was significantly increased on day $14(92.4 \% \pm 6.2$, $p<0.01$; Fig. 2A). Bleeding levels showed a similar increase during the experimental gingivitis challenge (day 14: $29.2 \% \pm 11.6, p<0.01$; Fig. $2 \mathrm{~B}$ ). At the end of the postchallenge "resolution phase," after 7 days of oral hygiene routines, dental plaque and bleeding levels returned back to expression levels at day 1 (day 21: plaque 62.1\% \pm 21.1 , bleeding $11.9 \% \pm 5.5$ ). These clinical measurements confirm the absence, development, and resolution of gingival inflammatory processes during the trial.

\section{Quantification of oPMNs}

The oPMN counts during the experimental gingivitis challenge and the postchallenge "resolution phase" are 

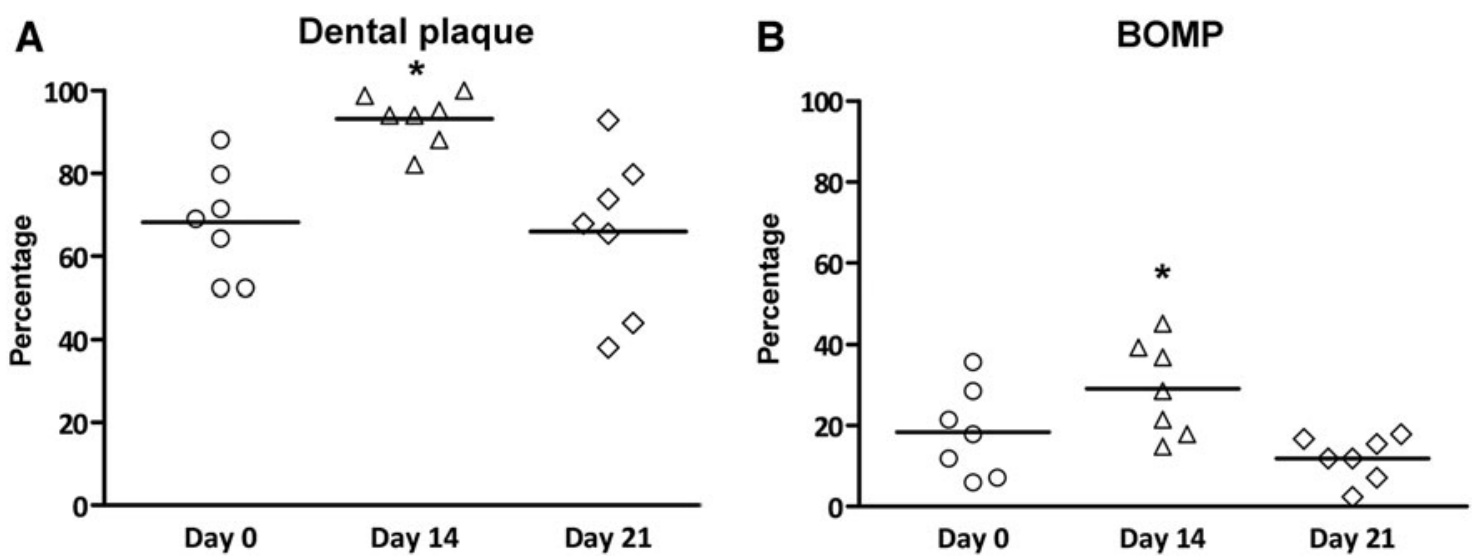

FIG. 2. Dental plaque accumulation and BOMP levels, stratified per time point $(n=7)$. The presence of dental plaque and BOMP were evaluated at six sites of the buccal and lingual aspects of the present teeth. Percentages were calculated by dividing the number of sites with dental plaque or BOMP by the total number of sites. The mean dental plaque percentages (A) were induced during the experimental gingivitis challenge and were significantly increased at day 14 compared with day 1 . The mean BOMP percentages (B) were also increased at day 14. Dots represent subjects, means are shown. *Comparison to the start of the experimental gingivitis challenge (day 1), Repeated measures analysis of variance, ${ }^{*} p<0.01$. BOMP, bleeding on marginal probing.

presented in Figure 3. The oPMN numbers increased by $26 \%$ in the current study group ( $n=41, p=0.03$, data not shown). No trend for increased oPMN numbers was observed for the seven subjects at day 14 (mean $0.5 \pm 0.6 \times 10^{7}, p=0.52$ ). During the postchallenge "resolution phase," the oPMN counts reduced slightly at day 21 (mean $0.4 \pm 0.2 \times 10^{7}$ ), but was not different compared with day 1 .

\section{Individual variation in oPMN transcriptome exceeds the variation induced by experimental gingivitis}

First, a multidimensional scaling (MDS) analysis was performed to explore the gene profiles of oPMNs from the subjects over time, during the trial. The MDS plots show clusters of gene responses per subject. The variation in the transcriptome per time point was less pronounced (Fig. 4). These results indicate that the oPMN transcriptome show a distinctive profile between individuals, which is less related to the different time points of the experimental gingivitis model.

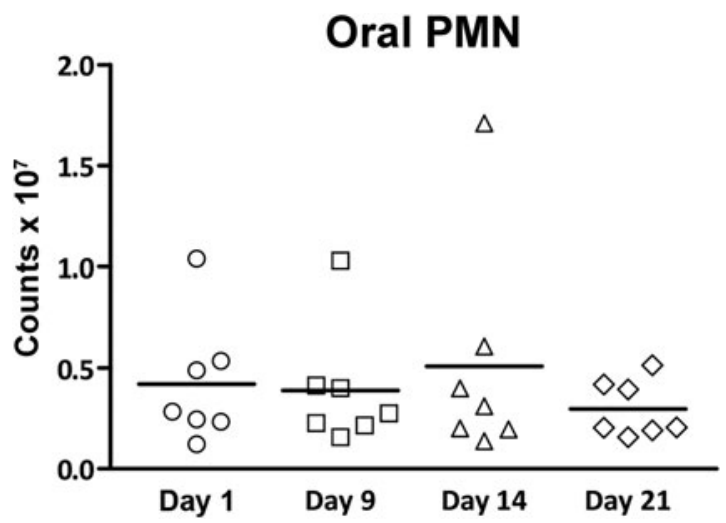

FIG. 3. Total oPMN yield, stratified per time point during the trial $(n=7)$. For the purpose of this study, seven subjects with comparable oPMN numbers were selected for further analysis. Dots represent individual subjects, means are shown.

\section{Distinct dynamics in oPMN transcriptomic responses} during experimental gingivitis

Differential expression analysis revealed that out of the 14,881 expressed genes, a total of 1165 genes to be significantly different $(p<0.01)$ in at least one time point during the trial, in comparison to the expression levels at day 1 (Fig. 5). A summary of the total number of DEGs, stratified per cluster can be found in Table 1. Detailed analysis of the oPMNs' transcriptional responses revealed two main clusters of genes associated with the experimental gingivitis challenge (day 9 and 14). Clusters consisted of a selected group of DEGs showing an initial response at day 9 only (total 256 DEGs; 161 upregulated, 95 downregulated) and another selected group of DEGs containing late response genes at day 14 only (total 221 DEGs; 124 upregulated, 97 downregulated). Most of the late responding DEGs, already showed a weak induction at day 9 and were almost completely reversed at day 21 .

An additional smaller group of persistently responding DEGs (both at day 9 and 14) was observed during the experimental gingivitis challenge (total 53 DEGs; 21 upregulated, 32 downregulated). These persistently responding DEGs showed a similar trend as the two main clusters and were almost completely reversed at day 21 . Furthermore, one additional cluster was found for the postchallenge "resolution phase" (day 21) containing a group of DEGs that were significantly changed compared with day 1 (total 524 DEGs; 164 upregulated, 360 downregulated), showing a weak induction during the experiment.

\section{Canonical pathway analysis demonstrate time-resolved responses of oPMNs during experimental gingivitis}

Canonical pathway analysis of the three main clusters and an overview of the top 10 canonical pathways stratified per time point can be found in Table 2. In total, $256 \mathrm{DEG}$ form a cluster of initial response (day 9), and showed involvement in interleukin (IL) signaling pathways, reactive oxygen species 

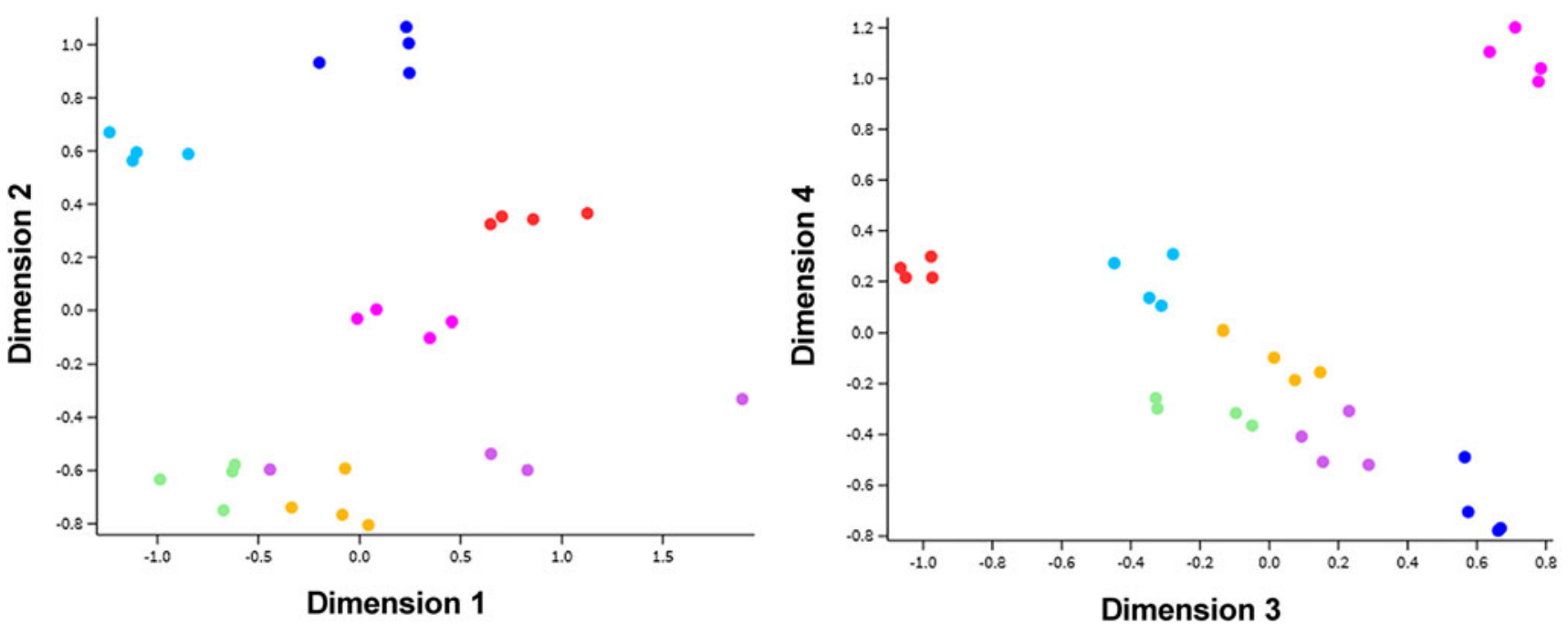

FIG. 4. MDS analysis plots of seven subjects during the experimental gingivitis trial. To evaluate potential clustering of the oPMN expression pattern MDS plots were generated. Colored dots represent the expression pattern of individual subject at one of the four time points. Samples showed grouping of subjects, whereas no obvious grouping was found per time point. Furthermore, less variances were observed when deeper dimensions were analyzed. MDS, multidimensional scaling.

(ROS) processes, and granulocyte adhesion and diapedesisrelated pathways. Energy-related pathways, like mitochondrial dysfunction and oxidative phosphorylation also showed a temporal activation at day 9 , which disappeared at day 14 and 21.

Late responding DEGs (day 14) consisted of 221 DEGs, and mainly showed activation of signaling pathways and phagosome formation as part of the phagocytosis process. The phagosome formation pathway was also significantly altered at day 9. This canonical pathway therefore consists of DEGs with persistently upregulated DEGs (at day 9 and 14), however, the phagosome formation pathway did not belong to the top 10 canonical pathways of the initial response
DEGs. At day 21, 524 DEGs were mainly involved in epithelial adherence signaling and other miscellaneous related signaling pathways.

\section{Discussion}

This study aimed to identify (1) the transcriptional responses of the oPMNs, and (2) whether changes at the gene expression level occur in relation to the development and resolution of gingival inflammation (e.g., gingivitis). We prospectively identified changes in the oPMN transcriptome of seven individuals at multiple time points. To the best of our knowledge, this study reports for the first time, the oPMN

A

B

C
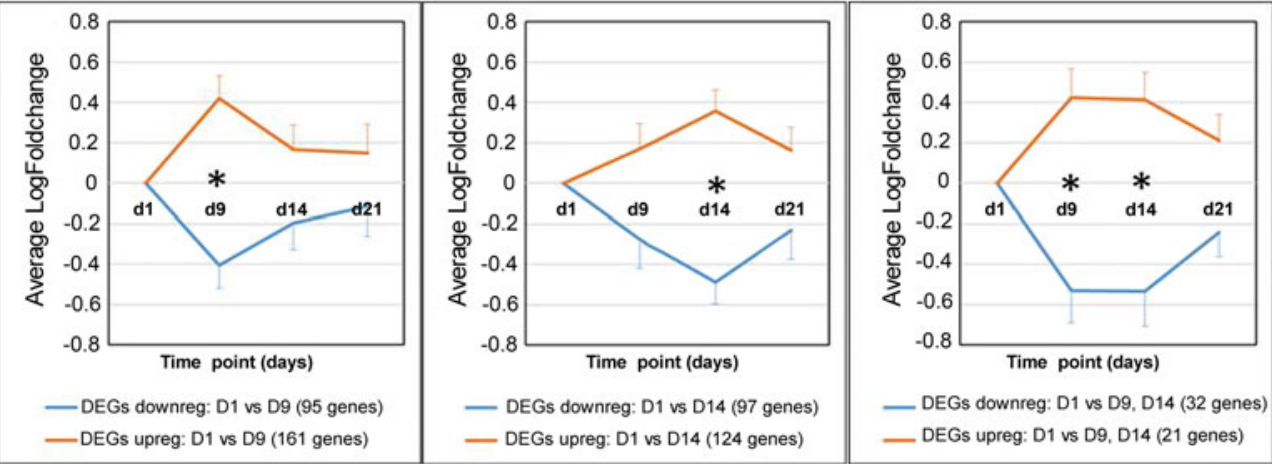

D

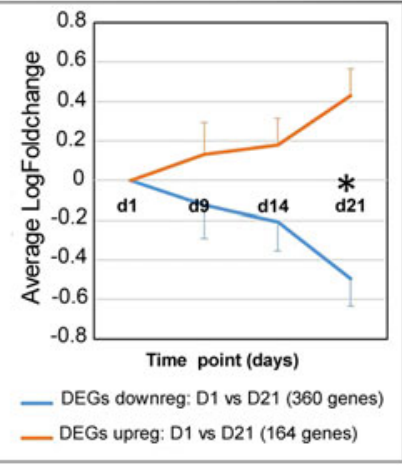

FIG. 5. Clusters of DEG. Analyses of 1165 DEGs revealed the presence of four main clusters $(p<0.01)$. Clusters contained significantly altered (upregulated or downregulated) DEGs at a specific time point. Three main clusters were observed during the experimental gingivitis challenge, containing a specific group of DEGs showing an early response at day 9 (A), another group of DEGs that were late responding genes at day $14(\mathbf{B})$, and a smaller cluster of persistently altered DEGs during the experimental challenge at both days [(C), day 9 and 14]. Most of the late responding DEGs at day 14, already show a weak induction at day 9 and are almost completely reversed at day 21 . Persistently upregulated DEGs (day 9 and 14) showed a similar trend and were almost completely reversed at day 21. An additional cluster of DEGs was found showing a significant response post-challenge at day 21 only (D), with a weak induction during the experimental gingivitis challenge. DEG, differentially expressed gene. 
Table 1. Number of Differentially Expressed Genes Stratified Per Cluster During the Experimental Gingivitis Trial

\begin{tabular}{lclr}
\hline \#DEGs (total) & \#DEG (cluster) & \multicolumn{1}{c}{ DEG cluster specific response (time point) } \\
\hline 530 & 256 & Initial responsive DEGs (day 9) & See Figure 5A \\
& 221 & Late responsive DEGS (day 14) & See Figure 5B \\
524 & 53 & Persistently responsive DEGs (day 9 and 14) & See Figure 5C \\
& & Responsive DEGs (day 21) & See Figure 5D \\
\hline
\end{tabular}

A total of 1165 significantly altered DEGs were found with a log fold change threshold of $\geq 2(p<0.01)$. Detailed analysis revealed three main clusters of DEGs based on each time point. An additional cluster containing persistently responsive DEGs was found during the experimental gingivitis trial. The expression pattern of the DEGs stratified per time point is presented in Figure 5.

DEG, differentially expressed gene.

transcriptome dynamics during the initiation and resolution of gingival inflammation in an experimental gingivitis model.

The human experimental gingivitis model is a valuable tool to study early oral inflammatory mechanisms. The results of our study showed that in the absence of a strong increase in oPMN numbers, several clusters of altered gene expression profiles presented during the whole trial. This suggests that oPMNs actively participate in response to the changes that occur within the oral ecosystem. Notably, this study observed several altered pathways during the early

Table 2. Overview of the Top Ten Canonical Pathways Stratified Per Time Point, Compared with Gene Expression Levels at Day One

\begin{tabular}{|c|c|c|c|c|c|}
\hline Day & Rank & Canonical pathway & $\begin{array}{c}\text { Day } 9 \\
256 \text { DEGs }\end{array}$ & $\begin{array}{c}\text { Day } 14 \\
221 \text { DEGs }\end{array}$ & $\begin{array}{c}\text { Day } 21 \\
524 \text { DEGs }\end{array}$ \\
\hline \multirow[t]{10}{*}{9} & 1 & Mitochondrial dysfunction & 3.3 & - & - \\
\hline & 2 & Phenylethylamine degradation I & 3.2 & - & - \\
\hline & 3 & Oxidative phosphorylation & 3.0 & - & - \\
\hline & 4 & Sirtuin signaling pathway & 2.9 & - & - \\
\hline & 5 & IL-17A signaling in gastric cells & 2.6 & - & - \\
\hline & 6 & IL-6 signaling & 2.6 & - & - \\
\hline & 7 & IL-7 signaling & 2.6 & - & - \\
\hline & 8 & Superoxide radicals degradation & 2.5 & - & - \\
\hline & 9 & Granulocyte adhesion and diapedesis & 2.5 & - & - \\
\hline & 10 & Agranulocyte adhesion and diapedesis & 2.5 & - & - \\
\hline \multirow[t]{10}{*}{14} & 1 & SUMOylation pathway & 0.7 & 4.8 & - \\
\hline & 2 & IL-8 signaling & 1.1 & 3.3 & - \\
\hline & 3 & IL-17A signaling in fibroblasts & 0 & 3.2 & - \\
\hline & 4 & Cholecystokinin/gastrin-mediated signaling & 1.7 & 3.0 & - \\
\hline & 5 & Glioma invasiveness signaling & 2 & 2.9 & - \\
\hline & 6 & ILK signaling & 0.4 & 2.7 & - \\
\hline & 7 & JAK/Stat signaling & 0 & 2.6 & - \\
\hline & 8 & Unfolded protein response & 0.9 & 2.4 & - \\
\hline & 9 & Actin nucleation by ARP-WASP complex & 0.9 & 2.4 & - \\
\hline & 10 & Phagosome formation & 2.4 & 2.4 & - \\
\hline \multirow[t]{10}{*}{21} & 1 & EIF2 signaling & 0.6 & 0 & 4.4 \\
\hline & 2 & Circadian rhythm signaling & 0 & 0 & 3.8 \\
\hline & 3 & Estrogen receptor signaling & 0.8 & 0 & 3.4 \\
\hline & 4 & Molecular mechanisms of cancer & 0 & 0.6 & 3.4 \\
\hline & 5 & $\begin{array}{l}\text { FLT3 signaling in hematopoietic } \\
\text { Progenitor cells }\end{array}$ & 0 & 0.7 & 2.8 \\
\hline & 6 & HIPPO signaling & 0 & 0 & 2.8 \\
\hline & 7 & Regulation of eIF4 and p70S6K signaling & 0.8 & 0.5 & 2.7 \\
\hline & 8 & Notch signaling & 1.3 & 1.0 & 2.6 \\
\hline & 9 & Melanocyte development and pigmentation signaling & 0 & 0 & 2.5 \\
\hline & 10 & Epithelial adherence junction signaling & 0 & 0 & 2.4 \\
\hline
\end{tabular}

DEGs were analyzed using IPA suite (http://ingenuity.com). Values represent the log ratio gene expressions compared with gene expression levels at day 1. IPA software was used as an input for canonical pathway analysis. Table 1 shows an overview of the DEGs stratified per cluster, per time point. A total of 256 DEGs formed a cluster of initial responsive DEGs (day 9), and were associated with interleukin signaling pathways, reactive oxygen species processes, and granulocyte adhesion and diapedesis-related pathways. Late responding DEGs (day 14) consisted of 221 DEGs in total, and mainly showed activation of signaling pathways and phagosome formation related to the phagocytosis process. Of note is that "Phagosome formation" was also found significantly altered at day 9, but was not enlisted in the top 10 of the initial response DEGs. At day 21, 524 DEGs were mainly associated with epithelial adherence signaling and other signaling pathways.

IPA, Ingenuity Pathway Analysis. 
stages of gingivitis development (reflecting an acute response pattern), which was then followed by a different oPMN transcriptome profile when gingival inflammation persisted (reflecting more toward chronicity).

A similar observation was previously observed, when PMNs from the blood circulation were treated with endotoxin (Calvano et al., 2005; de Kleijn et al., 2012). Altered transcriptomic profiles were observed in vivo when human subjects were administered with a single dose of endotoxin. Similar to the current study, these authors also observed changes related to early inflammatory responses that were followed by a recovery phase as shown by the observed changes in gene expression levels. In addition, extended PMN survival and regulation of various inflammatory responses were also observed.

For the current study, multiple clusters of DEGs were observed in oPMNs that included altered gene expressions associated with both pro- and anti-inflammatory pathways, and immunogenic pathways. Interestingly, a specific cluster of DEGs essential to the "resolution phase" was noticed, from which many of the affiliated IL and cytokine regulators were only different when the gene expressions from day 21 were compared with day 9 or 14 , but were comparable to the gene expressions at day 1 , the start of the experimental gingivitis challenge. This indicates that oPMNs can not only participate as acute responders during the initiation and the resolution of gingival inflammation, but also that the biological effects are reversible when subjects reinitiated their oral hygiene routines after refraining for a period of 2 weeks.

In general, when oral hygiene routines are abstained for a longer period of time, dental plaque accumulation occurs both along and in the submarginal area of the gingiva. The dental plaque maturation, "aging," and thickening of the oral microbial biofilm leads to a shift in the microbial composition (van der Veen et al., 2016; Volgenant et al., 2016), with more Gram-negative and anaerobic species taking advantage of the possible changes that occur within the oral ecosystem (Marsh and Zaura, 2017).

These microorganisms tend to illicit more responsiveness of the immune system (Tietze et al., 2006), which was also observed in our study. Even though the scope of this study did not include the identification of microbial species and their specific shifting pattern during the experimental gingivitis challenge, we presume that this also has occurred within our test subjects, resulting in the observed altered transcriptomic profiles from oPMNs. We isolated the oPMNs with extreme care from oral rinses, as was described before (Rijkschroeff et al., 2016, 2017). Morphologically, our sample preparations consisted of $\geq 80 \%$ oPMNs, which was also observed in our previous studies.

Nevertheless, the isolated mRNA could possibly be contaminated with mRNA from epithelial cells, since with the development of gingival inflammation other expected alterations include soft tissue changes of the gingival epithelium, like thinning of the sulcular epithelium, proliferation of pocket epithelium, and formation of rete ridges.

In response to the changes of the oral ecosystem, pathological dilation of the blood vessels also occurs with possibly new formation of capillaries of the gingival vasculature (Zoellner et al., 2002). This allows for an increased migration of PMNs and other immune cells into the gingival tissues, gingival crevices, and subsequently into the oral cavity (Dutzan et al., 2016; Landzberg et al., 2015; Moutsopoulos and Konkel, 2018). To account for the given nature of fluctuating oPMN numbers in oral inflammation, the current study investigated the transcriptome profiles from a selected group of individuals with comparable oPMN numbers. By selecting a group of individuals with a similar oPMN response in numbers, the results from this study may have also led to a more comparable transcriptome profile of the seven included study participants which is a limitation of this study.

It was previously observed that the transcriptomic profile of oPMNs from periodontitis patients were different from healthy controls, showing extended oPMN survival (Lakschevitz et al., 2013). Therefore, different transcriptome profiles may have presented more obviously when for example high responding individuals were compared with low responders in our study population. Another limitation is the low number of donors for the current study. In that respect, this study is to be regarded as a pilot study. By identifying DEGs related to the presented top 10 altered pathways at days 9,14 , and 21 , these data need to be considered as initial findings that need further confirmation.

Each studied time point within the trial showed similarities of the overall transcriptional profile, even though the specific pathways were not completely identical. At each separate time point, we observed multiple PMN-related functions within the top 10 altered pathways. Additionally, many IL signaling pathways were altered during the different time points, supporting the oPMNs' role in immune modulation.

As an example, IL-8 signaling was altered at day 14, which among other is involved in ROS production of PMNs (Chapple and Matthews, 2007). The number one pathway altered at day 14 of the experimental gingivitis challenge was SUMOylation pathway, which relates to the regulation of innate immune responses (Hannoun et al., 2016). SUMOylation is important in the posttranslational modifications involved in various cellular processes, which is very likely to occur also for oPMNs that are highly activated after 14 days of gingivitis development (Hay, 2005; Mahajan et al., 1997).

Additionally, our results also showed transcription-related pathways that were altered during the experimental gingivitis challenge (day 9: \#4 Sirtuin signaling pathway; day 14: \#1 SUMOylation, \#7 JAK/Stat signaling), whereas translationrelated pathways were altered postchallenge in the "resolution phase" (day 21: \#1 EIF2 signaling, \#7 Regulation of EIF4 and p70S6K signaling). A previous study showed that the transcriptome profile of PMN precursor cells correlated with the proteome profile of PMN granule subsets (Rorvig et al., 2013). Whether the transcriptome changes observed in the current study will also reflect the oPMNs' protein development and subsequent activation status, needs to be further investigated.

In general, the altered pathways found in the current study indicate broad activity of oPMNs that have migrated from the blood circulation into oral connective and epithelial tissues and subsequently are in a hostile bacteria-rich oral environment. Nowadays, more emerging evidence indicate that PMNs from the blood circulation are not only involved in antimicrobial functions such as phagocytosis and NET formation, but can also contribute to immunomodulatory functions. It was previously suggested that not only crosstalk occurs between PMNs and other immune cells, but also with epithelial cells (DiStasi and Ley, 2009; Kolaczkowska and Kubes, 2013; Mayadas et al., 2014; Szabady and 
McCormick, 2013). This allows for PMNs to maintain a homeostatic balance between production and turnover on site, while adapting to the local environmental challenges (Borregaard, 2010; Mayadas et al., 2014).

Based on our findings, we speculate that the oPMNs also play an immunomodulatory role within the gingival epithelium barrier, the gingival crevice, and subsequently in the oral cavity. We propose that a possible "feedback loop" evaluates oPMN homeostasis under resting and inflammatory conditions, through microbial surveillance and by crosstalk with epithelial cells and other immune cells.

\section{Conclusions}

Our observations suggest that oPMNs play a key role in the development of gingival inflammation and resolution. With this pilot study we report altered gene expressions affecting multiple pathways during the initiation and resolution of experimentally induced gingivitis. The observed altered gene expressions indicate their involvement as inflammatory mediators of the oral ecosystem. It changes the traditional view of oPMNs that were traditionally regarded as terminally differentiated granulocytes with a specific set of armories. As key regulators of the innate immune system, these transcriptionally active effectors are able to modulate oral inflammatory processes, possibly through microbial surveillance, crosstalk and other antimicrobial functions.

We contribute to renewed insights of the contribution of oPMNs in oral health equilibrium. However, in silico testing of each of the pathways would be a first step to deepen our knowledge on the vital role of oPMNs in maintaining oral health. Furthermore, our results could potentially be used to identify future biomarkers of oral disease activity, which could help identifying patients in various inflammatory disease processes.

\section{Author Contributions}

P.R. contributed to the design, data acquisition, analysis, interpretation of the data, and wrote the article. T.S. contributed to the data acquisition, analysis, interpretation of the data, and drafted the article. M.C. and L.V. contributed to data analysis, interpretation of the data, and critical revision of the article. B.G.L. and E.A.N. contributed to the conception, design, and interpretation of the data, and critical revision of the article. All authors have read and gave their approval for the article after significant intellectual contributions to the final version.

\section{Acknowledgments}

The authors thank N.A.M. Rosema for coordinating the clinical study "Exploring the Dynamics of the Oral Ecosystem During Experimental Gingivitis." The authors would like to express their sincere gratitude to S. Imangaliyev, I.D.C. Jansen, I. Persoon, C.M. Semeins, and D.S.L. Tjia for their help in collection of the samples. Furthermore, they would like to thank L. van Twillert for running the DESeq2 analysis.

\section{Author Disclosure Statement}

The authors declare they have no conflicting financial interests.

\section{Funding Information}

The Departments of Periodontology and Preventive Dentistry have been supported by a grant from the University of Amsterdam for research into the focal point " Oral Infections and Inflammation." The study was financed in part by the Academic Center for Dentistry Amsterdam Dental Research BV (ADR). Academic Center for Dentistry Amsterdam Dental Research BV has received funding from TI Food and Nutrition, a public/private partnership on precompetitive research in food and nutrition. Funding of this original research did not affect nor did it play a role in the conception of the study, the design, data acquisition, data analyses, and interpretation of the data.

\section{Supplementary Material}

Supplementary Figure S1

Supplementary Figure S2

\section{References}

Aboodi GM, Goldberg MB, and Glogauer M. (2011). Refractory periodontitis population characterized by a hyperactive oral neutrophil phenotype. J Periodontol 82, 726-733.

Bender JS, Thang H, and Glogauer M. (2006). Novel rinse assay for the quantification of oral neutrophils and the monitoring of chronic periodontal disease. J Periodontal Res 41, 214-220.

Borregaard N. (2010). Neutrophils, from marrow to microbes. Immunity 33, 657-670.

Calvano SE, Xiao W, Richards DR, et al. (2005). A networkbased analysis of systemic inflammation in humans. Nature 437, 1032-1037.

Chapple IL, and Matthews JB. (2007). The role of reactive oxygen and antioxidant species in periodontal tissue destruction. Periodontol 2000 43, 160-232.

de Kleijn S, Kox M, Sama IE, et al. (2012). Transcriptome kinetics of circulating neutrophils during human experimental endotoxemia. PLoS One 7, e38255.

Delima AJ, and Van Dyke TE. (2003). Origin and function of the cellular components in gingival crevice fluid. Periodontol 2000, 31, 55-76.

DiStasi MR, and Ley K. (2009). Opening the flood-gates: How neutrophil-endothelial interactions regulate permeability. Trends Immunol 30, 547-556.

Dutzan N, Konkel JE, Greenwell-Wild T, and Moutsopoulos NM. (2016). Characterization of the human immune cell network at the gingival barrier. Mucosal Immunol 9, 11631172.

Hannoun Z, Maarifi G, and Chelbi-Alix MK. (2016). The implication of SUMO in intrinsic and innate immunity. Cytokine Growth Factor Rev 29, 3-16.

Hay RT. (2005). SUMO: A history of modification. Mol Cell $18,1-12$.

Hirschfeld J. (2019). Neutrophil subsets in periodontal health and disease: A mini review. Front Immunol 10, 3001.

Kolaczkowska E, and Kubes P. (2013). Neutrophil recruitment and function in health and inflammation. Nat Rev Immunol 13, 159-175.

Lakschevitz FS, Aboodi GM, and Glogauer M. (2013). Oral neutrophil transcriptome changes result in a pro-survival phenotype in periodontal diseases. PLoS One 8, e68983.

Landzberg M, Doering H, Aboodi GM, Tenenbaum HC, and Glogauer M. (2015). Quantifying oral inflammatory load: 
Oral neutrophil counts in periodontal health and disease. J Periodontal Res 50, 330-336.

Lie MA, Timmerman MF, van der Velden U, and van der Weijden GA. (1998). Evaluation of 2 methods to assess gingival bleeding in smokers and non-smokers in natural and experimental gingivitis. J Clin Periodontol 25, 695-700.

Löe H, Theilade E, and Jensen SB. (1965). Experimental gingivitis in man. J Periodontol 36, 177-187.

Mahajan R, Delphin C, Guan T, Gerace L, and Melchior F. (1997). A small ubiquitin-related polypeptide involved in targeting RanGAP1 to nuclear pore complex protein RanBP2. Cell 88, 97-107.

Marsh PD, and Zaura E. (2017). Dental biofilm: Ecological interactions in health and disease. J Clin Periodontol 44 Suppl $18, \mathrm{~S} 12-\mathrm{S} 22$.

Matthews JB, Wright HJ, Roberts A, Ling-Mountford N, Cooper PR, and Chapple IL. (2007). Neutrophil hyperresponsiveness in periodontitis. J Dent Res 86, 718-722.

Mayadas TN, Cullere X, and Lowell CA. (2014). The multifaceted functions of neutrophils. Annu Rev Pathol 9, 181-218.

Moonen CGJ, Hirschfeld J, Cheng L, Chapple ILC, Loos BG, and Nicu EA. (2019). Oral neutrophils characterized: Chemotactic, phagocytic, and neutrophil extracellular trap (NET) formation properties. Front Immunol 10, 635.

Moutsopoulos NM, and Konkel JE. (2018). Tissue-specific immunity at the oral mucosal barrier. Trends Immunol 39, 276-287.

Rijkschroeff P, Jansen ID, van der Weijden FA, Keijser BJ, Loos BG, and Nicu EA. (2016). Oral polymorphonuclear neutrophil characteristics in relation to oral health: A cross-sectional, observational clinical study. Int J Oral Sci 8, 191-198.

Rijkschroeff P, Loos BG, and Nicu EA. (2017). Impaired polymorphonuclear neutrophils in the oral cavity of edentulous individuals. Eur J Oral Sci 125, 371-378.

Rorvig S, Ostergaard $\mathrm{O}$, Heegaard $\mathrm{NH}$, and Borregaard $\mathrm{N}$. (2013). Proteome profiling of human neutrophil granule subsets, secretory vesicles, and cell membrane: Correlation with transcriptome profiling of neutrophil precursors. J Leukoc Biol 94, 711-721.

Silness J, and Löe H. (1964). Periodontal disease in pregnancy. II. Correlation between oral hygiene and periodontal condition. Acta Odontol Scand 22, 121-135.

Silvestre-Roig C, Hidalgo A, and Soehnlein O. (2016). Neutrophil heterogeneity: Implications for homeostasis and pathogenesis. Blood 127, 2173-2181.

Stejskal S, Koutna I, and Rucka Z. (2010). Isolation of granulocytes: Which transcriptome do we analyse-neutrophils or eosinophils? Folia Biol 56, 252-255.

Subrahmanyam YV, Yamaga S, Prashar Y, et al. (2001). RNA expression patterns change dramatically in human neutrophils exposed to bacteria. Blood 97, 2457-2468.

Szabady RL, and McCormick BA. (2013). Control of neutrophil inflammation at mucosal surfaces by secreted epithelial products. Front Immunol 4, 220.

Tietze K, Dalpke A, Morath S, Mutters R, Heeg K, and Nonnenmacher C. (2006). Differences in innate immune responses upon stimulation with gram-positive and gramnegative bacteria. J Periodontal Res 41, 447-454. van der Veen MH, Volgenant CM, Keijser B, Ten Cate JB, and Crielaard W. (2016). Dynamics of red fluorescent dental plaque during experimental gingivitis - a cohort study. J Dent 48, 71-76.

Van der Velden U. (2009). The Dutch periodontal screening index validation and its application in The Netherlands. J Clin Periodontol 36, 1018-1024.

Van der Weijden GA, Timmerman MF, Nijboer A, Lie MA, and Van der Velden U. (1993). A comparative study of electric toothbrushes for the effectiveness of plaque removal in relation to toothbrushing duration. Timerstudy. J Clin Periodontol 20, 476-481.

Volgenant CM, Hoogenkamp MA, Buijs MJ, Zaura E, Ten Cate JM, and van der Veen MH. (2016). Red fluorescent biofilm: The thick, the old, and the cariogenic. J Oral Microbiol 8, 30346.

Wahaidi VY, Kowolik MJ, Eckert GJ, and Galli DM. (2011). Endotoxemia and the host systemic response during experimental gingivitis. J Clin Periodontol 38, 412-417.

White PC, Chicca IJ, Cooper PR, Milward MR, and Chapple IL. (2016). Neutrophil extracellular traps in periodontitis: A web of intrigue. J Dent Res 95, 26-34.

World Medical Association. (2013). World Medical Association Declaration of Helsinki: Ethical principles for medical research involving human subjects. JAMA 310, 2191-2194.

Zoellner H, Chapple CC, and Hunter N. (2002). Microvasculature in gingivitis and chronic periodontitis: Disruption of vascular networks with protracted inflammation. Microsc Res Tech 56, 15-31.

Address correspondence to:
Patrick Rijkschroeff
Department of Periodontology
Academic Centre for Dentistry Amsterdam (ACTA)
University of Amsterdam (UVA)
and Vrije Universiteit Amsterdam (VU)
Amsterdam 1081 LA
The Netherlands

E-mail: p.rijkschroeff@acta.nl

\begin{aligned} & \multicolumn{1}{c}{ Abbreviations Used } \\ ACTA & $=$ Academic Center for Dentistry Amsterdam \\ BOMP & $=$ bleeding on marginal probing \\ DEG & $=$ differentially expressed gene \\ DPSI & $=$ Dutch Periodontal Screening Index \\ $\mathrm{IL} & =$ interleukin \\ IPA & $=$ Ingenuity Pathway Analysis \\ MDS & $=$ multidimensional scaling \\ NET & $=$ neutrophil extracellular trap \\ oPMN & $=$ oral polymorphonuclear neutrophil \\ PMN & $=$ polymorphonuclear neutrophil \\ ROS & $=$ reactive oxygen species \\ SD & $=$ standard deviation \end{aligned}




\section{Supplementary Data}

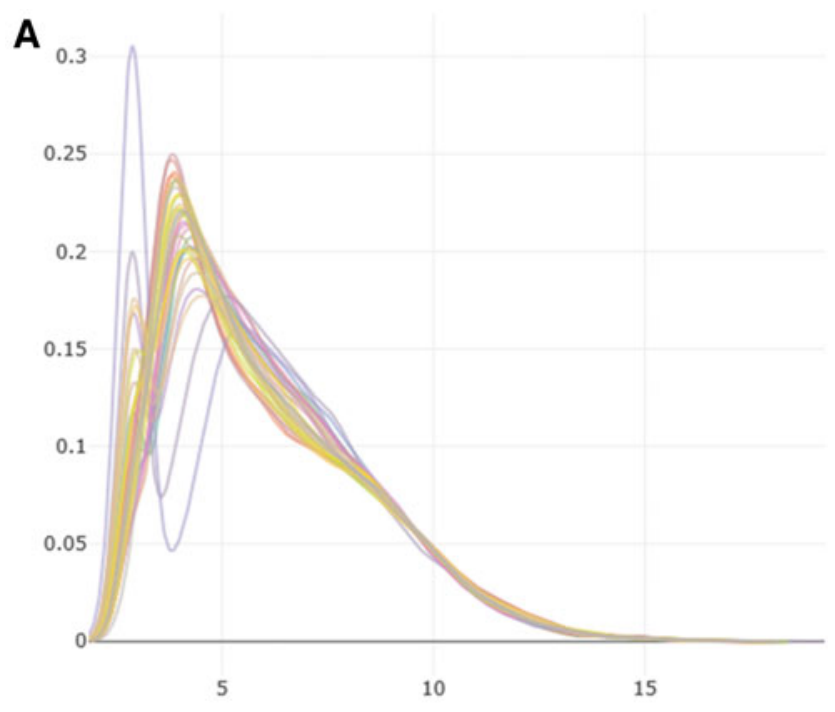

B

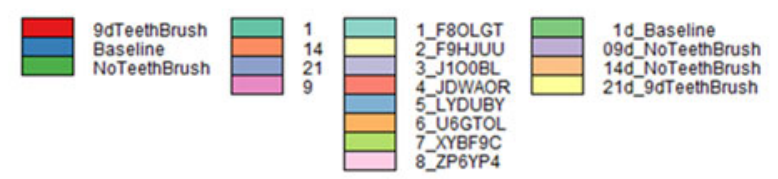

SUPPLEMENTARY FIG. S1. Exploratory analysis of 32 samples originating from eight subjects. An intensity distribution (A) and a sample distance matrix + heatmap (B) were made to evaluate the presence of deviant PMN expression patterns. These revealed two outliers (*sample 9 and *sample 11) belonging to one subject in particular. The deviating samples did not contribute to more differentially expressed genes, although the model corrected for subject differences when the raw data were normalized. For this reason, samples originating from this subject were removed from further analyses. PMN, polymorphonuclear neutrophil.
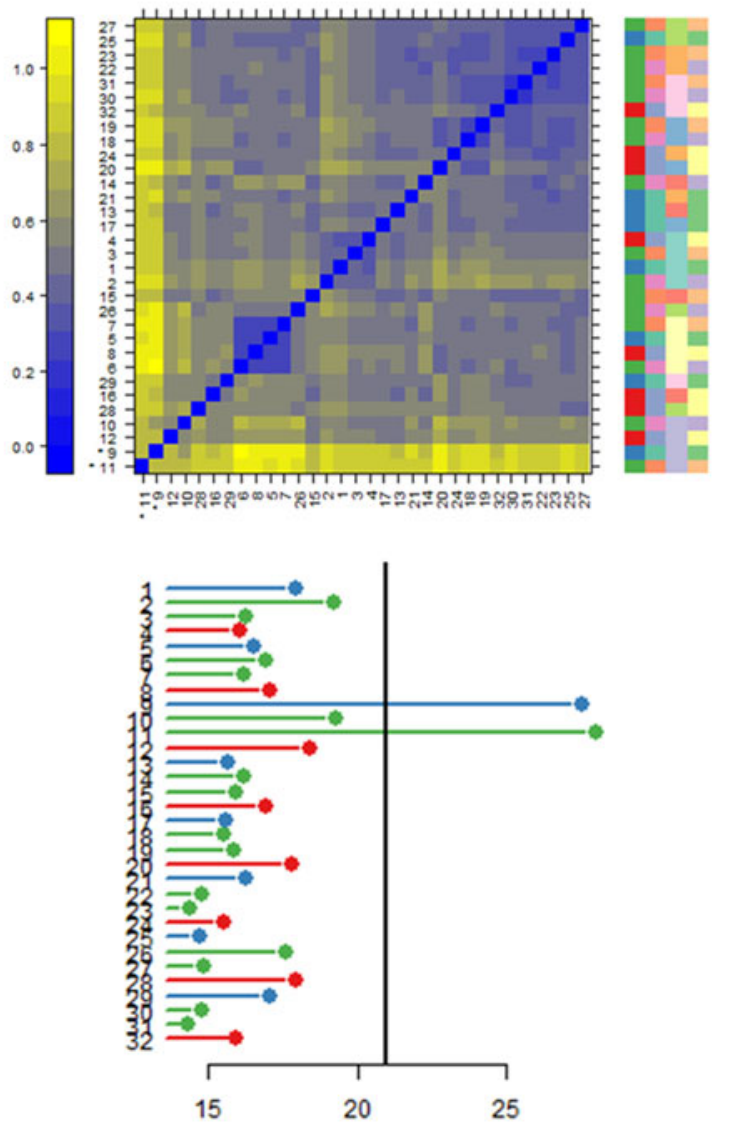
A

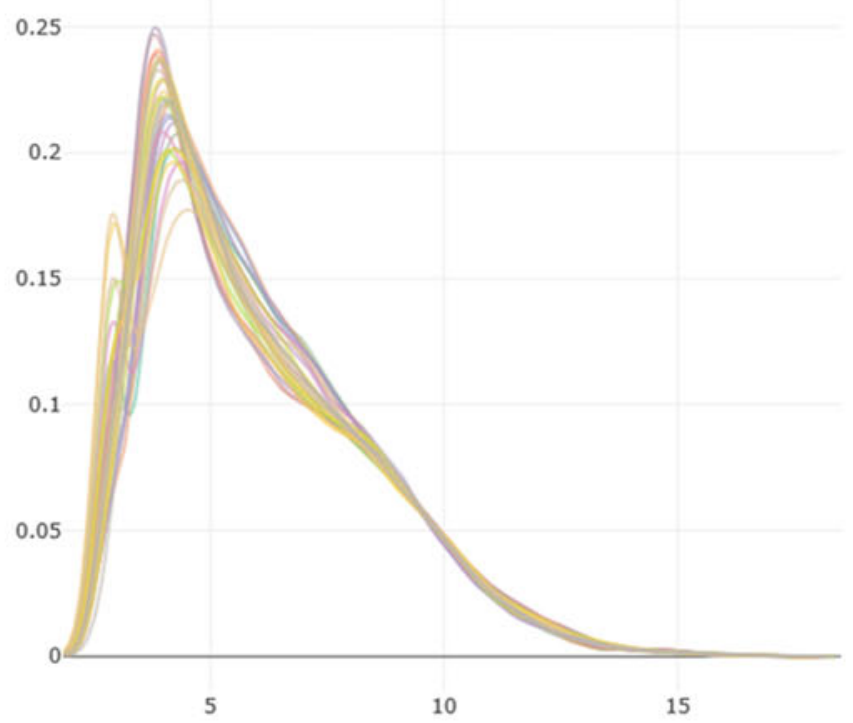

B
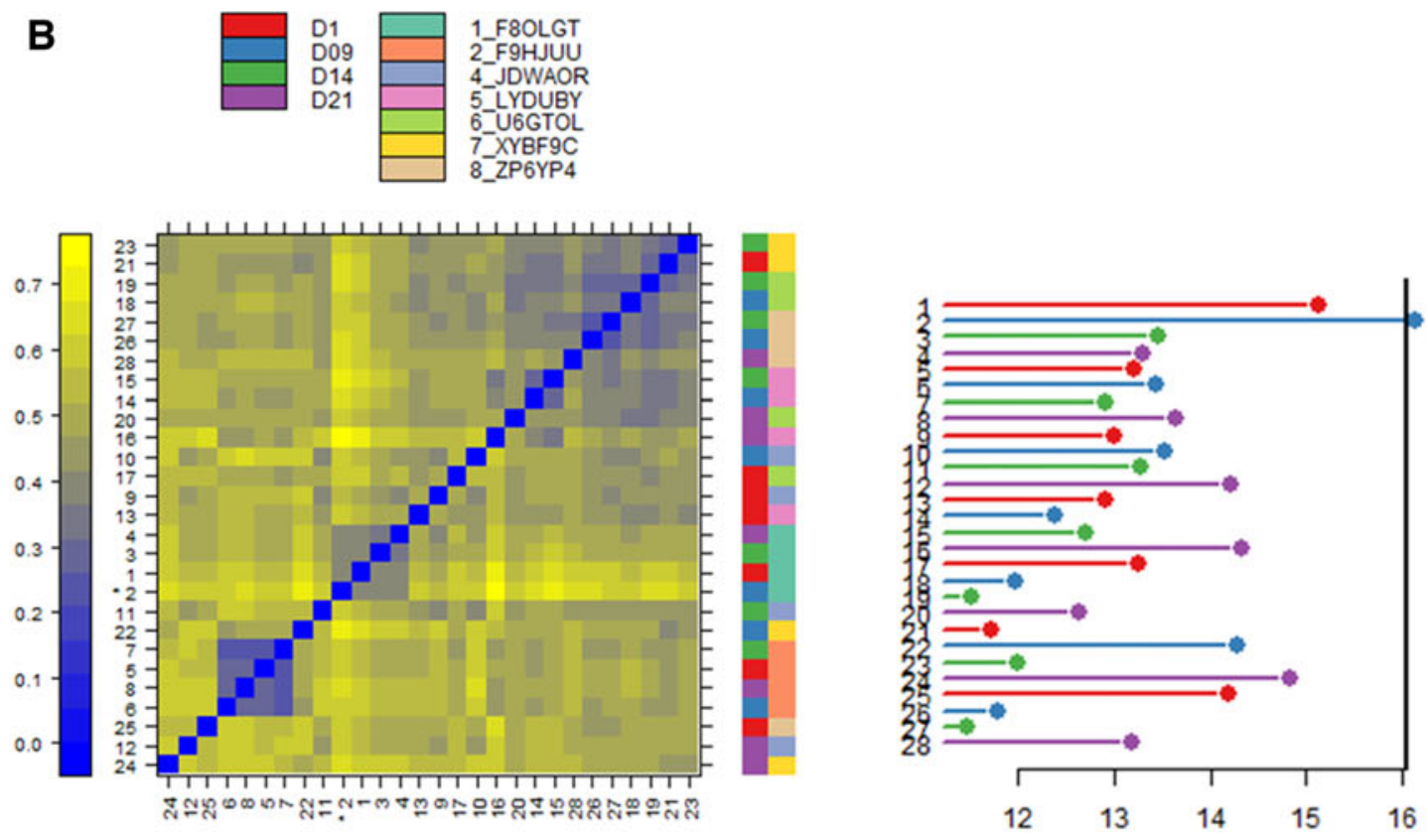

\section{Raw sequencing depth}

\section{Normalized sequencing depth}
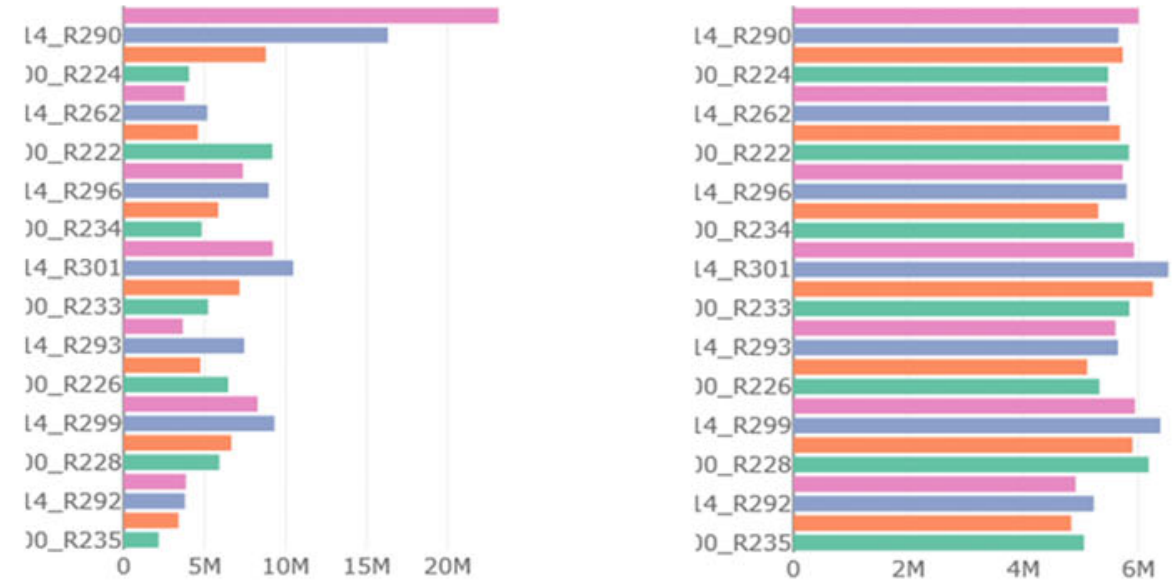

SUPPLEMENTARY FIG. S2. Differential analysis of 28 samples originating from seven subjects. An intensity distribution (A) and a sample distance matrix + heatmap (B) were made to evaluate the presence of deviant PMN expression patterns. No obvious outliers or deviating samples were observed. Sequencing Depth analysis (C) was performed to evaluate the data after normalization. 